\title{
Brisure De Symétrie Et Nombres De Lychrel
}

\author{
Latifa Ait abida, Aymane Sghiar, Iliès sghiar, M. sghiar \\ 9 Allée capitaine J. B. Bossu, 21240, Talant, France
}

\begin{abstract}
We show that the existence of the numbers of lychrel is due to a symmetry breaking in the space of numbers.

Résumé: Nous montrons que l'existence des nombres de lychrel est due à une brisure de symétrie dans l'espace des nombres.
\end{abstract}

Keywords: Nombre de lychrel, Théorie des nombres, conjecture de Syracuse.

\section{Introduction}

Un nombre de Lychrel est un nombre naturel qui ne peut pas former de nombre palindrome lorsqu'il est soumis au processus itératif qui consiste à l'additionner au nombre formé de l'inversion de ses chiffres en base 10. Le nom «Lychrel» a été inventé par Wade VanLandingham : il s'agit d'une quasi-anagramme du nom de sa fiancée, Cheryl. Les nombres de Lychrel étaient considérés comme des nombres théoriques. On n'en connaît aucun, bien que de nombreux nombres soient suspectés. Le plus petit nombre suspecté d'être de Lychrel est 196. C'est donc lui qui a attiré le plus d'attention. John Walker a commencé la recherche le 12 août 1987 avec un programme écrit en $\mathrm{C}$ qui effectuait les itérations et qui vérifiait si le nombre était un palindrome. Cela dura presque 3 ans, et finit (comme cela lui était demandé) le 24 mai 1990 avec le message suivant :

Point d'arrêt atteint après 2415836 itérations.

Le nombre possède 1000000 chiffres. Ainsi 196 a donc atteint un nombre à un million de chiffres après 2415836 itérations sans parvenir à un palindrome. Walker publia ses recherches et en 1995, Tim Irvin utilisa un superordinateur et atteint un nombre de 2 millions de chiffres en seulement 3 mois sans trouver de palindrome. Jason Doucette poursuivit la lancée et atteint 12,5 millions de chiffres en mai 2000. Wade VanLandingham utilisa le programme de Jason Doucette pour atteindre 13 millions de chiffres, un record publié dans Yes Mag, magazine des sciences pour enfant au Canada. Le $1^{\mathrm{er}}$ mai 2006, VanLandingham atteignit la barre des 300 millions de chiffres (avec une moyenne d'un million de chiffres tous les 5 ou 7 jours). Malgré cela aucun palindrome n'a été trouvé.Voir: Jean Paul Delahaye, http://www.lifl.fr/ jdelahay/IAGL/Conjectures.pdf et http://www.p196.org/. D'autres candidats comme 879, 1997 et 7059 ont aussi été longuement testés par la même méthode de brute force en atteignant aussi plusieurs millions d'itérations sans trouver le moindre palindrome.

Ce problème évoque la fameuse conjecture de Syracuse, nommée aussi problème de Collatz démontré dans [M. Sghiar] : Dans cette conjecture, N est divisé par 2 s'il est pair, et remplacé par $3 \mathrm{~N}+1$ s'il est impair. Ce procédé finit par donner 1.

Par des techniques quasi similaires à la preuve de la conjecture de Syracuse [M. Sghiar], le But de cette article est de montrer que les nombres de lychrel existent bel et bien, et que leur existence est due à une brisure de symétrie.

\section{Idée De La Preuve}

L'idée de la preuve est inspirée de la brisure de symétrie qui joue un rôle important en physique.

on va assimiler les nombres entiers à des particules d'un ensemble $\mathbb{N}$ que l'on complète par un ensemble

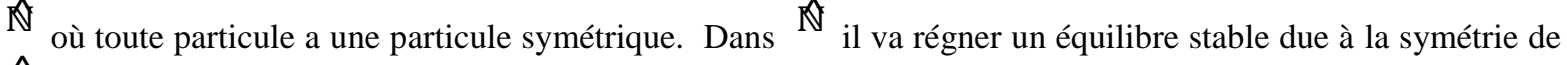

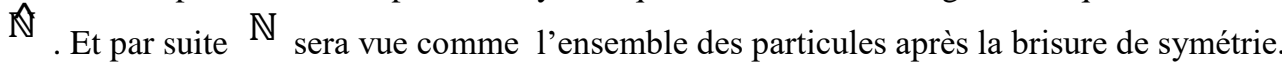

Cette brisure de symétrie va rendre l'ensemble des particules instables et causera une sorte d' explosion qui va éjecter tous les éléments symétriques des particules de $\mathbb{N} \mid \mathbb{N}$ qu'on notera $(\mathbb{N} \mid \mathbb{N})^{s}$.

On remarque que le pourcentage des éléments de $(\mathbb{N} \mid \mathbb{N})^{s}$ dans l'ensemble $\mathbb{N}^{\mathbb{2}}$ est de $10 / 100$, ce qui correspondra à la moyenne des éléments des nombres de lychrel.

\section{Définitions}

Posons $x^{s}$ le le symétrique du nombre $\mathrm{x}$ : Exemple : $123^{s}=321,1000^{s}=0001$. 
Un nombre $\mathrm{x}$ est dit Palindrome si $x^{s}=x$.

Remarque : $001 \notin \mathbb{N}$, d'où la définition :

Posons $\mathbb{N}=\mathbb{N} \cup \mathbb{N}^{s}$ où $\mathbb{N}^{s}=\left\{x^{s}, x \in \mathbb{N}\right\}$.

Soit $\phi$ la fonction de $\mathbb{N}_{\text {sur }} \mathbb{N}$ par : $\phi(x)=x+x^{s}$.

Un nombre entier x est dit un nombre de Lychrel si $\forall i \in \mathbb{N} \quad \phi^{i}(x)_{\text {n'est pas un nombre palindrome. }}$

Soit $\psi$ la fonction définie de $\mathbb{N}$ sur $\mathbb{Z}$ par: $\psi(x)=x-x^{s}$.

Il est claire qu'un nombre $\mathrm{x}$ est Palindrome si et seulement si $\psi(x)=0$.

\section{Enoncée des conjectures}

\section{Conjecture du palindrome inévitable :}

Tout nombre entier soumis de manière répétée à l'opération renversement-addition finit par produire un palindrome.

Conjecture du devenir infini de 196 :

Le nombre 196 soumis à l'opération renversement-addition ne produit jamais un palindrome.

Théorème 1:

\section{Existence des nombres de lychrels}

$\forall x \in \mathbb{N}, \quad \exists i \in \mathbb{N} /(\psi-I)\left(\phi^{i}(x)\right)=-\phi^{i}(x)$

\section{Preuve :}

En assimilant tout nombre x de $\mathbb{N}$ à une particule, la fonction $\phi$ induit la force $\phi-I$ (où I est l'identité) : $(\phi-I)(x)=\phi(x)-x=x^{s}$

De même la fonction $\psi$ induit la force $\psi-I$ (où I est l'identité) : $(\psi-I)(x)=\psi(x)-x=-x^{s}$.

Donc les forces $\phi-I$ et $\psi^{-I}$ exercent des forces opposées sur chaque particule x, il est donc intéressant d'étudier l'action de la fonction $\psi$ sur $\mathbb{N}$.

Remarquons d'abord que contrairement à la fonction $\phi$ qui a tendance à pousser toute particule vers la droite, l'action de $\psi$ est chaotique : certaines particules sont poussées vers la droite alors que d'autres sont tirées vers la gauche, d'où l'intérêt de voir si le mouvement agité des particules va se stabiliser.

Effectivement, comme $\psi(x)=x-x^{s}$, alors $\psi\left(x^{s}\right)=-\psi(x)$, de sorte que la résultante des forces induites par $\psi$ sur chaque particule est nulle. Il s'ensuit que la résultante des forces induites par $\phi$ sur chaque particule est deux fois l'identité. Il en résulte que dans l'orbite de $\mathrm{x}: O(x)=\left\{\phi^{i}(x), i \in \mathbb{N}\right\}$, $\forall x \in \mathbb{N}, \exists i \in \mathbb{N} /(\phi-I)\left(\phi^{i}(x)\right)=\phi^{i}(x)$ puisque $\quad \forall x \in \mathbb{N}, \exists i \in \mathbb{N} /(\psi-I)\left(\phi^{i}(x)\right)=-\phi^{i}(x)$, soit $\psi\left(\phi^{i}(x)\right)=0$

\section{Corollaire 1:}

Pour tout élément $\mathrm{x}$ dans $\mathbb{N}, \exists i \in \mathbb{N} / \psi\left(\phi^{i}(x)\right)=0$

\section{Corollaire 2:}

Dans $\mathbb{N}$, il existe des nombres x tels que : $\psi\left(\phi^{i}(x)\right) \neq 0 \quad \forall i \in \mathbb{N}$. 


\section{Preuve :}

En effet, en enlevant les particules symétriques des particules de la forme $k 10^{l}$ on brise la symétrie (ou l'équilibre), donc les particules se trouvent libre et se déplacent vers la droite (sous l'action de $\phi$ )sans s'arrêter, d'où le résultat.

En posant $\mathbb{Z}=\{ \pm x, x \in \mathbb{N}\}$, on peut prolonger $\Psi$ à $\mathbb{Z}$ par :

$$
\Psi(-x)=-\Psi(x)
$$

\section{Corollaire 3:}

Pour tout élément x dans $\mathbb{Z}, \exists i \in \mathbb{N} / \psi^{i}(x)=0$

Preuve :

Analogue à celle du corollaire 1 ci-dessus en considérant $\psi$ au lieu de $\phi$.

Corollaire 4:

Dans $\mathbb{N}$, il existe des nombres x tels que $\psi^{i}(x) \neq 0 \quad \forall i \in \mathbb{N}$. (Exemple $x=69325$ ).

Preuve :

Analogue à celle du corollaire 2 ci-dessus.

Pour $x=69325$, on trouve: $\psi^{7}(69325)=2178$ et $\psi^{9}(69325)=-2178$, on en déduit que : $\forall i \in \mathbb{N} / \psi^{i}(69325) \not \neq 0$

\section{Remarques:}

i- Les particules symétriques des particules de $\mathbb{N} \mid \mathbb{N}$ représentent un pourcentage moyen de 10/100 dans $\mathbb{N}$, et cela correspond exactement au pourcentage moyen des nombres de Lychrel suspects. Ce qui explique que les nombres de lychrels peuvent être assimilées à des particules libres à cause de la brisure de symétrie.

ii- Les nombres de lychrel peuvent s'écrire sous la forme: $\sum \epsilon_{i, j} \phi^{i}(x)$ où $\epsilon_{i, j} \in\{-1,1\}$ et x un élément de $(\mathbb{N} \mid \mathbb{N})^{s}$, autrement dit, comme en mécanique quantique, tout nombre de lychrel est un niveau d'énergie ou représente un état qui est une combinaison des états $\phi^{i}(x)$.

\section{Exemple:}

Voici les premiers nombres de lychrels suspects :

196, 295, 394, 493, 592, 689, 691, 788, 790, 879, 887, 978, 986, 1 495, 1 497, 1 585, 1 587, 1 675, 1677,1765 , $1767,1855,1857,1945,1947,1997 \ldots$

$$
\begin{aligned}
& \text { On a: } \\
& \begin{aligned}
196 & =\phi^{5}(10)+\phi^{0}(20)=\phi^{4}(20)+\phi^{0}(20) \\
493 & =\phi^{4}(30)+\phi^{0}(160) \\
592 & =\phi^{4}(50)+\phi^{0}(300) \\
689 & =\phi^{4}(90)+\phi^{0}(400) \\
887 & =\phi^{4}(80)+\phi^{0}(40) \\
978 & =\phi^{4}(100)+\phi^{0}(170) \\
295 & =\phi^{4}(80)-\phi^{4}(50)-\phi^{0}(260) \\
394 & =\phi^{4}(80)-\phi^{4}(30)-\phi^{0}(120)
\end{aligned}
\end{aligned}
$$


$691=\phi^{4}(80)-\phi^{4}(20)+\phi^{0}(20)$

$986=\phi^{1}(50)+\phi^{0}(1220)-\phi^{4}(90)$

$1495=\phi^{4}(70)+\phi^{0}(890)$

$1497=\phi^{4}(80)+\phi^{0}(650)$

$1585=\phi^{4}(100)+\phi^{1}(70)+\phi^{0}(700)$

$1587=\phi^{4}(80)+\phi^{0}(740)$

$1675=\phi^{1}(50)+\phi^{0}(1620)$

$1677=\phi^{1}(70)+\phi^{0}(1600)$

De même pour les autres nombres suspects (jusqu'au 1997).

\section{Références}

[1]. Jean-Paul Delahaye, http://www.lifl.fr/ jdelahay/IAGL/Conjectures.pdf

[2]. http://www.p196.org/

[3]. M. Sghiar, La relativité et la théorie des nombres, prépublication, https://hal.archives-ouvertes.fr/hal-01174146v4 